The Agriculturists 18(1):116-128(2020) ISSN 2304-7321 (Online), ISSN 1729-5211 (Print)

A Scientific Journal of Krishi Foundation

Indexed Journal

DOI: https://doi.org/10.3329/agric.v18i1.49464

\title{
Variability and Character Association Study in Morphological Traits of Tossa Jute (Corchorus olitorius L.)
}

\author{
Anusree Ghosh*, Bhabendra Kumar Biswas and Md. Arifuzzaman \\ Department of Genetics and Plant Breeding, Hajee Mohammad Danesh Science and Technology \\ University, Dinajpur 5200, Bangladesh
}

*Corresponding author and Email: anusreeghosh62@gmail.com

Received: 26 April 2020

Accepted: 25 June 2020

\begin{abstract}
Tossa jute (Corchorus olitorius L.) is the most important commercial crop of Bangladesh. Fifty-six genotypes of $\mathrm{F}_{2}$ generation were raised at Breeding Research Farm, Hajee Mohammad Danesh Science and Technology University, Dinajpur from April 2018 to August 2019 from the parental lines O-9897, O-795, JRO-524, Acc. 2381, Acc. 3423, Acc. 3438, Acc. 3533 and Acc. 3860. The parameters, plant height $(\mathrm{m})$, base diameter $(\mathrm{mm})$, green bark thickness $(\mathrm{mm})$, green bark weight without leaves $(\mathrm{g})$, stick weight (g), fiber strength (MPa), days to flowering, days to maturity and fiber weight/stick varied significantly among the genotypes. Significant productivity was found from Acc. 3860. Green bark weight without leaves exhibited highest genotypic and phenotypic co-efficient of variation followed by fiber weight and stick weight. The highest genetic advance as percentage of mean (44.38\%) was estimated against green bark weight without leaves suggest it as a prime character that enhanced fiber and stick yields. The Acc. 3860 resulted high production of fiber in hybrids. Considering the studied characters, the F2-generation of Acc. 3860 is comparatively batter than others. The research findings suggested that the F2-generation of Accession Acc. 3860 may produce desired segregates and therefore demands farther research in different environmental conditions and field trail.
\end{abstract}

Keywords: Tossa jute, morphological features, yield, correlation, path coefficient of yield contributing characters

\section{Introduction}

Bangladesh is known as 'Land of Golden Fiber' because of the jute production. Jute is one of the cheapest and the strongest of all-natural fibers and considered as fiber of the future. Current concern of climate change and environmental pollutions have newly realized the importance of jute. Improved and stress tolerant variety has long been demanding for future sustainability. The nature and magnitude of variability of a crop indicates the potentiality of breeding. Yield is regarded as a complex character which is influenced by many components or contributing traits both in positive and negative directions (Rabbani et al., 2012). For effective selection, information on the nature and magnitude of variation in the population, association of characters with yield and among themselves and extent of environmental influences on the expression of these characters are necessary. It then becomes necessary to partition the observed 
variability into its heritable and non-heritable components with the help of suitable genetic parameters.

Genetic variability forms the basic factor to be considered while making germplasm selection for crop improvement. Heritability indicates transmissibility of a character in future generations (Satheeshkumar and Saravanan, 2012). Selection based on only yield is often unwise. So, it is necessary to know the association between yield and yield components which may be determined by correlation analysis (Akhtar et al. 2011). The level of contribution of each yield component trait on yield into direct and indirect effects is determined by path coefficient analysis (Ahmadizadeh et al., 2011). Considering the above facts, Bangladesh needs high yielding jute cultivars that should be achieved through study of genetic variability and character association with some inbred lines and experimental hybrid derivatives of Tossa jute (Corchorus olitorius). The major aim of the study was to find out character association in experimental hybrid derivatives of and their parents of Tossa Jute. However, the objectives of the study were i) Assessment of diversity and similarities among the segregants; ii) Advancing the generation derived from the selected segregants

\section{Materials and Methods}

\subsection{Plant materials}

A set of 56 experimental hybrids of Tossa Jute from 8 Accessions/ Varieties have been raised at Plant Breeding Research Field of Department of Genetics and Plant Breeding, Hajee Mohammad Danesh Science and Technology University, Dinajpur.

\subsection{Experimental design and cultivation}

The seeds of the experimental materials were sown in well-prepared land in Randomized Complete Block Design with three replications. The experiment was seeded on 16 April 2018. The seeds of each genotype were sown in 3 rows of $3 \mathrm{~m}$ long. The rows were $30 \mathrm{~cm}$ apart with planting space of $5-7 \mathrm{~cm}$. The replication blocks were interspaced with $60 \mathrm{~cm}$. The genotypes were assigned randomly to each plot within each block. The agronomic practice with fertilizer application were in accordance with normal recommendations.

The field experimental site was in Plant Breeding Research Field, Hajee Mohammad Danesh Science and Technology University, Dinajpur. It is situated $25^{\circ} 13 \mathrm{~N}$ latitude and $88^{\circ} 23^{\prime} \mathrm{E}$ longitude at $37.5 \mathrm{~m}$ altitude, in the tropical climate zone, characterized by heavy rainfall during the month April to August and scanty rainfall during rest of the year; average temperatures were maximum $33{ }^{\circ} \mathrm{C}$ and minimum $12{ }^{\circ} \mathrm{C}$ and the average rainfall was $1745 \mathrm{~cm}$ in 2018. Major cultivation concerns were as follows:

\subsubsection{Land preparation}

The experimental plot was at a lower elevation with high water holding capacity. The land was prepared thoroughly with 3-4 ploughing and cross ploughing followed by harrowing and laddering.

\subsubsection{Fertilizer application}

The crop was fertilized with recommended doses of well decomposed cowdung (5t/ha) and fertilizers such as $100 \mathrm{~kg} /$ ha Urea, $25 \mathrm{~kg} / \mathrm{ha}$ TSP and $45 \mathrm{~kg} / \mathrm{ha}$ MOP respectively. The whole amount of TSP, MOP and half of the amount of urea were applied at final land preparation. The remaining half urea was applied in two equal installments after first and final weeding.

\subsubsection{Irrigation and drainage}

After germination of seed, the field was irrigated properly and, necessary soil moisture maintained throughout the crop period. A good drainage system was also ensured for immediate release of excess rainwater from the experimental field during the crop growing period.

\subsubsection{Intercultural operations}

Seeds were sown on 30 April 2018. Thinning and weeding were done twice after 15 and 35 
days of sowing to maintain uniform plant population. Necessary intercultural operations were performed for proper growth and development of the plants. Thinning and weeding were done simultaneously at the time of two top dressings of nitrogen. These practices were helpful to break the soil crust, to maintain uniform growth of plant population and to incorporate the nitrogen fertilizer (urea) into the soil, thus reducing the loss through denitrification. Proper control measures were taken against mite, nematode and stem rot of jute. The infested jute hairy caterpillars at larval and pupal stage were handpicked and destroyed. 2.2.5 Crop harvesting: The sample plants of individual plots were harvested for fiber yield at 120 days from the date of sowing, which is considered as the physiologically optimum age for quality fiber (Majumdar et. al., 2013). The crop was cut at the base with the help of sickle and kept separately. The other details of materials and methods are described in the relevant of the specific experiments.

\subsection{Duration and data recordings}

The experiment was conducted during the period from April 2018 to August 2019. The data on fiber yield and its attributes as bellow were recorded from 3 randomly selected plant of each genotypes from each replication after harvest i.e. 120 days of crop age: Plant height (m), base diameter $(\mathrm{mm})$, green bark thickness $(\mathrm{mm})$, green bark weight without leaves $(\mathrm{Kg})$, stick weight (g), fiber strength (MPa), days to flowering, days to maturity, fiber weight (g), and fiber weight / stick . The details of data recording were as below:

1. Plant Height (m): It was measured from the base to the tip of the main shoot of 5 randomly selected plants in meter and the mean was computed.

2. Base Diameter (mm): Average base diameter of 5 randomly selected plants was measured at the base of the stem in millimeter using slide calipers.

3. Green Bark Thickness (mm): The green bark thickness was measured in $\mathrm{mm}$. It was done by subtracting from basal diameter to core diameter. Core diameter was measured by pulling the basal bark of the stem.

4. Green Weight without Leaves (kg): Average green weight of previously selected plants without leaves was recorded after defoliation of leaves, which was seven days after harvest.

5. Stick Weight (g): Weight of sun-dried stick per $\mathrm{m} 2$ area was measured after drying and the mean was computed.

6. Fiber Strength (MPa): Fiber strength is the strongness of the fiber. Fiber strength was measured by tensile strength in the lab of Cotton Research, Training and Seed Multiplication Farm, Sadarpur, Dinajpur.

7. Days to $50 \%$ Flowering: Days to $50 \%$ flowering was determined by counting the numbers of days from emergence of seedlings up to appearance of $50 \%$ plants bearing flowers.

8. Day to $80 \%$ maturity: Number of days from sowing to the maturity of the $80 \%$ of the pods of whole plot.

9. Fiber Weight per plant (g): Weight of fiber derived from a plant that is measured by $\operatorname{gram}(\mathrm{g})$

10. Fiber weight / stick weight: It was calculated from the data of fiber weight and stick weight by direct dividing.

\subsection{Statistical analysis}

The analysis of variance (ANOVA) was estimated according to Panse and Sukhatme (1989), and Alam (2009). Simple correlation coefficient (r) among 11 important characters of Tossa Jute were estimated with following formula (Miller et al., 1958; Singh and Chaudhary, 1985, Alam, 2009, Rahman et al., 2009). Correlation co-efficient components of different yield attributes with grain yield were portioned in to components of direct and indirect effects by path co-efficient analysis followed by Singh and Chaudhary (1985) and Dabholkar (1992). The data were analyzed using the software package $\mathrm{R}$ of version 3.4.2 and Statistical Tool for Agricultural Research (STAR) Version: 2.0.1. of PNGB Division, 
International Rice Research Institute. The significant and non-significant differences among the genotypes for a character were estimated by Least Significant Difference (LSD) test.

\section{Results and Discussion}

\subsection{Variance among different phenotypic characters in Tossa jute}

The analysis of variance of ten phenotypic characters is presented in Table 2. According to the Table 2, significant differences revealed for all the parameters studied. Higher magnitude of variance was recorded in base diameter, green bark thickness and green bark weight without leaves. The co-efficient of variation $(\mathrm{CV})$ ranged from $1.56-15.11 \%$. The mean performance of fifty-six Tossa jute genotypes for eleven morphological characters are presented in Table 3 . The wide range of phenotypic performances variation exhibited in the genotypes for all the characters. The height of the jute plant varies from 2.57 meter to 3.76 meter with an average height 3.37 where the standard deviation was 0.23 . The lowest height was recorded for the hybrids of P6 x P5 and also the hybrids of P6 were comparatively of low height. The highest height was recorded for the P2 x P7 $(3.79 \mathrm{~m})$ followed by P5 x P8 $(3.75 \mathrm{~m})$. Five tallest among the parents and the hybrids were the crosses of P2, P3, P5 and P8. Among the parent height of the $\mathrm{P} 8$ was the tallest followed by $\mathrm{P}_{2}$. It was found that the height of the $F_{2}$ generation was taller than the parents. Islam (2019) noted range of jute height 1.5 to $3.7 \mathrm{~m}$ tall. Highest jute plant height $(444.33 \mathrm{~cm})$ was observed in case of olitorius (var. JRO 66) followed var. JRO 878 $(443.33 \mathrm{~cm})$ (Das and Kumar, 2012). They also noted that the low variability for jute was recorded in case of plant height. Ghosh et al. (2017) has classified 133 genotypes of jute in to 4 class, viz., below 1.00 , class $1.01-2.00$, class 2.01-3.00 and above 3.00. Among his studied genotypes plant height ranged from 2.11 [OIN 302 (India, Karnataka)] to $3.68 \mathrm{~m}$ [OIJ 245 (Nepal)].

Table 1. Characteristics of eight selected genotypes of tossa jute used as materials.

\begin{tabular}{|c|c|c|c|c|}
\hline $\begin{array}{l}\text { Sl. } \\
\text { No. }\end{array}$ & $\begin{array}{c}\text { Accessions/ } \\
\text { Varieties }\end{array}$ & Origin & $\begin{array}{c}\text { Parental } \\
\text { symbol }\end{array}$ & Other characteristics \\
\hline 01. & O-9897 & O-5 x BZ-5 & P1 & $\begin{array}{l}\text { Plant is full green, leaves are ovate, lanceolate, fruit } \\
\text { indehiscence in nature, seed bluish green in colour, } \\
\text { less photosensitive and late maturing. }\end{array}$ \\
\hline 02. & O-795 & $\begin{array}{l}\text { Uganda red } \\
\mathrm{x} \mathrm{O}-4\end{array}$ & $\mathrm{P} 2$ & $\begin{array}{l}\text { Plant reddish, leaves ovate, lanceolate, fruit } \\
\text { indehiscence in nature, seed bluish green in colour, } \\
\text { less photosensitive and late maturing }\end{array}$ \\
\hline 03. & JRO-524 & $\begin{array}{l}\text { Indian } \\
\text { origin }\end{array}$ & P3 & $\begin{array}{l}\text { Plant full green, leaves ovate, lanceolate, fruit } \\
\text { indehiscence and sticky in nature, seed bluish green } \\
\text { in colour, less photosensitive and late maturing. }\end{array}$ \\
\hline 04. & Acc. 2381 & $\begin{array}{l}\text { Local } \\
\text { collection }\end{array}$ & P4 & $\begin{array}{l}\text { Plant reddish, leaves ovate, lanceolate, seed bluish } \\
\text { green in colour. }\end{array}$ \\
\hline 05. & Acc. 3423 & $\begin{array}{l}\text { Local } \\
\text { collection }\end{array}$ & P5 & $\begin{array}{l}\text { Plant full green, leaves ovate, lanceolate, seed bluish } \\
\text { green in colour. }\end{array}$ \\
\hline 06. & Acc. 3438 & $\begin{array}{l}\text { Local } \\
\text { collection }\end{array}$ & P6 & $\begin{array}{l}\text { Plant reddish, leaves ovate, lanceolate seed bluish } \\
\text { green in colour. }\end{array}$ \\
\hline 07. & Acc. 3533 & $\begin{array}{l}\text { Local } \\
\text { collection }\end{array}$ & P7 & $\begin{array}{l}\text { Plant full green, leaves ovate, lanceolate, seed bluish } \\
\text { green in colour. }\end{array}$ \\
\hline 08. & Acc. 3860 & $\begin{array}{l}\text { Local } \\
\text { collection }\end{array}$ & P8 & $\begin{array}{l}\text { Plant reddish, leaves ovate, lanceolate, seed bluish } \\
\text { green in colour. }\end{array}$ \\
\hline
\end{tabular}


Table 2. Analysis of variance of phenotypic characters in tossa jute

\begin{tabular}{lcccc}
\hline $\begin{array}{l}\text { Sources of variation } \\
(\mathrm{df})\end{array}$ & $\begin{array}{c}\text { Replication } \\
(2)\end{array}$ & $\begin{array}{c}\text { Genotype } \\
(55)\end{array}$ & $\begin{array}{c}\text { Error } \\
110\end{array}$ & $\begin{array}{c}\mathrm{CV} \\
(\%)\end{array}$ \\
\hline Plant height $(\mathrm{m})$ & 13.68 & $0.16^{*}$ & 0.26 & 15.11 \\
Base diameter $(\mathrm{mm})$ & 51.15 & $1.90^{* *}$ & 0.16 & 2.48 \\
Green bark thickness $(\mathrm{mm})$ & 46.81 & $3.40^{* *}$ & 0.06 & 1.56 \\
Green weight without leaves $(\mathrm{Kg})$ & 50.13 & $1.13^{* *}$ & 0.08 & 9.35 \\
Fiber weight $(\mathrm{g})$ & 481.346 & $50919.36^{*}$ & 496.28 & 7.3 \\
Stick weight $(\mathrm{g})$ & 3.24 & $10925.80^{* *}$ & 150.18 & 4.63 \\
Fiber strength (MPa) & 10603.08 & $8462.25^{*}$ & 7348.99 & 14.46 \\
Days to flowering & 79.63 & $17.93^{*}$ & 20.08 & 5.82 \\
Days to maturity & 12.67 & $25.20^{*}$ & 25.75 & 4.52 \\
\hline
\end{tabular}

NB: $*$ and $* *$ indicates significant at $5 \%$ and $1 \%$ levels of probability, respectively.

Base diameter of the studied jute was ranged from $14.40 \mathrm{~mm}$ to $17.96 \mathrm{~mm}$. The highest diameter value was recorded for $\mathrm{P} 8 \mathrm{xP} 2$ followed by $\mathrm{P} 8 \mathrm{xP} 3$ and $\mathrm{P} 8 \mathrm{xP7}$. Apart from the generation of the P8, some hybrids of P1 also have shown good values of base diameter. Significant varietal performance on base diameter was also obtained by Pervin and Haque (2012); Islam (2007); Azad-ud-doula Prodhan et al., (2001) in jute and Hossain et al. (2011 and 2012) in Kenaf.

Green bark thickness ranges from $1.13 \mathrm{~mm}$ to $1.7 \mathrm{~mm}$ with a mean value of $1.5 \mathrm{~mm}$, the standard deviation was 1.7. Like the base diameter, the highest value was recorded for the hybrids of $\mathrm{P} 8$. The $\mathrm{P} 8 \mathrm{xP} 4$ has the height value followed by P8xP5> P8xP6. The lowest green bark thickness was reported in P5xP4. Pervine and Haque (2012) noted that ark thickness had highest positive direct effect on fibre yield per plant. These findings are in accordance with Ali (1984), Ahmed and Khatun (1997) and Islam et al., (2002).

The green weight without leaves varies from $2.22 \mathrm{Kg}$ to $4.50 \mathrm{Kg}$, i.e. the height value hybrid has it nearly double of the low values. Here all crosses of P8 were also recorded higher followed by the crosses of P1. The slandered deviation for the Green bark weight without leaves were recorded comparatively low 0.61 . Billah et al., (2015) recorded the fresh weight without leaves per plant the environmental mean and genotypic mean ranged from 223.3 to $240.6 \mathrm{~g}$ and 199.4 to $311.7 \mathrm{~g}$, respectively. This study also identified the genotypes C-2753 showed stability for fresh weight with leaves, without leaves and dry stick weight.

Fiber weight recorded minimum was 369.35 for P2xP1 followed by P7xP5, where the highest value for it was nearly 3 times for $\mathrm{P} 8 \mathrm{xP} 2$, all other P8 hybrids provided the heights value. Dry fiber weight ranged from $9.20-40.00 \mathrm{~g} /$ plant in the study by Biswas et al., (2018). It was found highest in accession 3730 which was followed by accessions 5086, 1076, 3724, 2966 and variety O-9897. Similar result was found in Annual Research Report 2016 of Bangladesh Jute Research Institute in different accession.

Stick weight also has a huge deviation, the ratio among the lowest and the highest is more than double. P4xP1 has the lowest production whereas P8xP3 has the height. Though the average value is 264.90 , and 46 hybrids are producing stick weight less than $3000 \mathrm{~g}$. The highest (10.74 t ha-1) weight of fresh stick was obtained from O-9897 and the lowest weight (7.20 t ha-1) by Sarker et al., (2012). Akter et al., (2010) and Nayak et al., (2009) reported considerable contribution of stick weight to the total divergence. 
Table 3. Mean performance of accessions/varieties of tossa jute for ten yield and yield contributing characters

\begin{tabular}{|c|c|c|c|c|c|c|c|c|c|c|}
\hline & $\begin{array}{l}\text { Plant } \\
\text { height } \\
(\mathrm{m})\end{array}$ & $\begin{array}{c}\text { Base } \\
\text { diameter } \\
(\mathrm{mm}) \\
\end{array}$ & $\begin{array}{c}\text { Green bark } \\
\text { thickness } \\
(\mathrm{mm})\end{array}$ & $\begin{array}{c}\text { Green weight } \\
\text { without } \\
\text { leaves }(\mathrm{Kg}) \\
\end{array}$ & $\begin{array}{c}\text { Fiber } \\
\text { weight } \\
(\mathrm{g}) \\
\end{array}$ & $\begin{array}{c}\text { Stick } \\
\text { weight }(\mathrm{g})\end{array}$ & $\begin{array}{c}\text { Fiber } \\
\text { strength } \\
(\mathrm{MPa})\end{array}$ & $\begin{array}{l}\text { Days to } \\
\text { flowering }\end{array}$ & $\begin{array}{l}\text { Days to } \\
\text { maturity }\end{array}$ & $\begin{array}{c}\text { Fiber } \\
\text { weight/ } \\
\text { Stick weight }\end{array}$ \\
\hline $\mathrm{P} 1 \times \mathrm{P} 2$ & 3.36 & 15.72 & 1.448 & 3.30 & 559.41 & 283.25 & 535.00 & 78.33 & 107.33 & 1.97 \\
\hline P1 x P3 & 3.53 & 16.34 & 1.638 & 3.40 & 652.43 & 310.29 & 588.33 & 77.00 & 110.00 & 2.10 \\
\hline P1 x P4 & 3.33 & 15.37 & 1.616 & 3.33 & 609.43 & 262.27 & 693.33 & 73.67 & 118.00 & 2.32 \\
\hline P1 x P5 & 3.41 & 14.63 & 1.630 & 2.55 & 517.41 & 247.92 & 506.67 & 76.00 & 109.67 & 2.09 \\
\hline P1 x P6 & 3.41 & 15.47 & 1.612 & 2.49 & 509.59 & 235.04 & 534.33 & 70.67 & 114.67 & 2.17 \\
\hline P1 x P7 & 3.52 & 15.52 & 1.437 & 2.57 & 610.56 & 237.42 & 495.33 & 79.33 & 117.00 & 2.57 \\
\hline P1 x P8 & 3.53 & 15.39 & 1.534 & 3.48 & 721.43 & 324.49 & 605.33 & 78.00 & 110.33 & 2.22 \\
\hline $\mathrm{P} 2 \times \mathrm{P} 3$ & 3.34 & 14.40 & 1.530 & 2.57 & 638.56 & 283.52 & 586.00 & 77.00 & 112.33 & 2.25 \\
\hline $\mathrm{P} 2 \times \mathrm{P} 4$ & 3.36 & 16.29 & 1.524 & 2.49 & 656.40 & 265.72 & 590.00 & 77.33 & 116.67 & 2.47 \\
\hline P2 x P5 & 3.43 & 15.24 & 1.445 & 2.56 & 623.81 & 256.44 & 586.00 & 79.00 & 106.33 & 2.43 \\
\hline $\mathrm{P} 2 \times \mathrm{P} 6$ & 3.56 & 15.66 & 1.435 & 3.44 & 551.41 & 242.73 & 576.33 & 79.33 & 113.00 & 2.27 \\
\hline $\mathrm{P} 2 \times \mathrm{P} 7$ & 3.76 & 15.18 & 1.423 & 3.17 & 520.69 & 210.45 & 631.67 & 75.67 & 113.33 & 2.47 \\
\hline $\mathrm{P} 2 \times \mathrm{P} 8$ & 3.40 & 16.69 & 1.442 & 3.15 & 581.96 & 238.49 & 663.33 & 75.33 & 111.00 & 2.44 \\
\hline $\mathrm{P} 3 \times \mathrm{P} 4$ & 3.44 & 16.24 & 1.548 & 3.01 & 447.62 & 210.66 & 675.33 & 80.00 & 112.00 & 2.12 \\
\hline P3 x P5 & 3.41 & 15.30 & 1.640 & 3.47 & 560.44 & 242.54 & 661.00 & 79.33 & 111.33 & 2.31 \\
\hline P3 x P6 & 3.51 & 16.35 & 1.579 & 3.51 & 629.48 & 256.74 & 507.67 & 78.33 & 112.33 & 2.45 \\
\hline P3 x P7 & 3.48 & 16.75 & 1.544 & 3.59 & 677.55 & 290.74 & 547.67 & 71.67 & 111.00 & 2.33 \\
\hline P3 x P8 & 3.60 & 17.14 & 1.637 & 3.48 & 710.29 & 309.20 & 554.33 & 74.33 & 116.00 & 2.30 \\
\hline P4 x P5 & 3.53 & 15.75 & 1.426 & 2.63 & 560.04 & 224.61 & 633.33 & 72.33 & 110.67 & 2.49 \\
\hline P4 x P6 & 3.66 & 16.47 & 1.553 & 2.28 & 440.04 & 228.46 & 639.67 & 77.33 & 107.67 & 1.93 \\
\hline P4 x P7 & 3.65 & 15.54 & 1.544 & 3.44 & 464.34 & 213.57 & 621.33 & 77.67 & 117.67 & 2.17 \\
\hline P4 x P8 & 3.54 & 15.47 & 1.532 & 4.49 & 709.52 & 325.54 & 681.00 & 80.00 & 113.00 & 2.18 \\
\hline P5 x P6 & 3.22 & 15.15 & 1.567 & 2.92 & 693.45 & 292.39 & 563.33 & 75.33 & 114.33 & 2.37 \\
\hline P5 x P7 & 3.55 & 16.60 & 1.538 & 2.49 & 503.24 & 220.39 & 610.00 & 81.67 & 115.33 & 2.28 \\
\hline P5 x P8 & 3.71 & 15.32 & 1.554 & 2.34 & 625.62 & 228.52 & 560.67 & 77.00 & 111.00 & 2.74 \\
\hline P6 x P7 & 3.43 & 16.61 & 1.535 & 3.44 & 649.46 & 285.65 & 568.00 & 76.00 & 113.00 & 2.27 \\
\hline P6 x P8 & 3.06 & 14.50 & 1.455 & 2.59 & 412.53 & 228.04 & 529.33 & 76.00 & 107.00 & 1.81 \\
\hline P7 x P8 & 3.00 & 16.39 & 1.632 & 3.46 & 631.54 & 280.44 & 543.33 & 73.33 & 112.33 & 2.25 \\
\hline $\mathrm{P} 2 \times \mathrm{P} 1$ & 3.45 & 15.67 & 1.450 & 2.69 & 369.35 & 216.48 & 605.33 & 76.33 & 109.67 & 1.71 \\
\hline P3 x P1 & 3.33 & 17.27 & 1.551 & 2.59 & 507.19 & 227.45 & 574.67 & 77.67 & 109.67 & 2.23 \\
\hline
\end{tabular}




\begin{tabular}{|c|c|c|c|c|c|c|c|c|c|c|}
\hline & $\begin{array}{c}\text { Plant } \\
\text { height } \\
(\mathrm{m})\end{array}$ & $\begin{array}{c}\text { Base } \\
\text { diameter } \\
(\mathrm{mm}) \\
\end{array}$ & $\begin{array}{c}\text { Green bark } \\
\text { thickness } \\
(\mathrm{mm})\end{array}$ & $\begin{array}{c}\text { Green weight } \\
\text { without } \\
\text { leaves }(\mathrm{Kg})\end{array}$ & $\begin{array}{c}\text { Fiber } \\
\text { weight } \\
(\mathrm{g})\end{array}$ & $\begin{array}{c}\text { Stick } \\
\text { weight }(\mathrm{g})\end{array}$ & $\begin{array}{c}\text { Fiber } \\
\text { strength } \\
(\mathrm{MPa})\end{array}$ & $\begin{array}{l}\text { Days to } \\
\text { flowering }\end{array}$ & $\begin{array}{l}\text { Days to } \\
\text { maturity }\end{array}$ & $\begin{array}{c}\text { Fiber } \\
\text { weight/ } \\
\text { Stick weight }\end{array}$ \\
\hline P3 x P2 & 3.48 & 16.34 & 1.571 & 3.29 & 417.94 & 246.27 & 568.00 & 80.00 & 109.33 & 1.70 \\
\hline $\mathrm{P} 4 \times \mathrm{P} 1$ & 3.46 & 16.30 & 1.552 & 3.15 & 496.20 & 203.44 & 583.00 & 77.33 & 115.00 & 2.44 \\
\hline $\mathrm{P} 4 \times \mathrm{P} 2$ & 3.32 & 15.24 & 1.446 & 3.33 & 581.36 & 223.42 & 648.33 & 80.67 & 113.67 & 2.60 \\
\hline P4 x P3 & 3.22 & 16.26 & 1.552 & 3.19 & 569.33 & 258.74 & 609.33 & 81.00 & 117.33 & 2.20 \\
\hline P5 x P1 & 3.28 & 15.29 & 1.548 & 3.21 & 579.49 & 265.60 & 583.33 & 81.33 & 113.33 & 2.18 \\
\hline P5 x P2 & 3.16 & 16.63 & 1.439 & 2.28 & 484.61 & 217.45 & 656.67 & 73.33 & 115.33 & 2.23 \\
\hline $\mathrm{P} 5 \times \mathrm{P} 3$ & 3.38 & 14.46 & 1.536 & 2.75 & 627.25 & 241.44 & 653.33 & 77.00 & 108.67 & 2.60 \\
\hline P5 x P4 & 3.52 & 15.63 & 1.621 & 3.50 & 729.42 & 300.55 & 516.67 & 76.00 & 115.33 & 2.43 \\
\hline P6 x P1 & 2.73 & 16.46 & 1.138 & 2.56 & 457.22 & 243.55 & 510.00 & 77.67 & 117.67 & 1.88 \\
\hline $\mathrm{P} 6 \times \mathrm{P} 2$ & 2.65 & 16.16 & 1.480 & 2.53 & 422.96 & 227.45 & 604.33 & 77.33 & 109.33 & 1.86 \\
\hline P6 x P3 & 3.22 & 15.81 & 1.549 & 2.63 & 398.42 & 204.47 & 636.33 & 79.67 & 112.33 & 1.95 \\
\hline P6 x P4 & 3.23 & 16.45 & 1.535 & 2.34 & 567.46 & 213.53 & 597.67 & 73.67 & 113.67 & 2.66 \\
\hline P6 x P5 & 2.57 & 16.52 & 1.436 & 2.29 & 551.60 & 230.22 & 615.00 & 79.33 & 110.33 & 2.40 \\
\hline P7 x P1 & 3.53 & 16.36 & 1.625 & 2.55 & 459.21 & 210.19 & 620.00 & 75.67 & 109.33 & 2.18 \\
\hline $\mathrm{P} 7 \times \mathrm{P} 2$ & 3.38 & 16.42 & 1.448 & 3.38 & 553.71 & 245.34 & 448.33 & 76.00 & 111.67 & 2.26 \\
\hline P7 x P3 & 3.27 & 16.32 & 1.262 & 2.22 & 479.57 & 221.21 & 578.67 & 77.67 & 109.00 & 2.17 \\
\hline P7 x P4 & 3.20 & 15.52 & 1.650 & 2.51 & 496.53 & 225.52 & 701.67 & 74.00 & 111.67 & 2.20 \\
\hline P7 x P5 & 3.21 & 15.34 & 1.624 & 2.48 & 393.44 & 204.28 & 588.00 & 76.33 & 113.67 & 1.93 \\
\hline P7 x P6 & 3.51 & 15.96 & 1.530 & 3.56 & 741.19 & 323.57 & 549.33 & 78.33 & 114.00 & 2.29 \\
\hline P8 x P1 & 3.38 & 16.37 & 1.553 & 2.45 & 444.53 & 203.46 & 569.33 & 79.00 & 111.33 & 2.18 \\
\hline $\mathrm{P} 8 \times \mathrm{P} 2$ & 3.55 & 17.96 & 1.675 & 4.50 & 914.36 & 427.43 & 650.33 & 74.00 & 111.33 & 2.14 \\
\hline P8 x P3 & 3.34 & 17.39 & 1.632 & 4.41 & 890.59 & 440.47 & 643.33 & 78.67 & 114.67 & 2.02 \\
\hline $\mathrm{P} 8 \times \mathrm{P} 4$ & 3.44 & 17.30 & 1.743 & 4.05 & 864.94 & 415.21 & 616.67 & 77.33 & 115.00 & 2.08 \\
\hline P8 x P5 & 3.40 & 17.14 & 1.729 & 4.30 & 840.63 & 391.69 & 607.67 & 75.67 & 115.67 & 2.15 \\
\hline P8 x P6 & 3.47 & 16.97 & 1.718 & 4.02 & 846.66 & 367.53 & 595.33 & 76.00 & 111.67 & 2.30 \\
\hline $\mathrm{P} 8 \times \mathrm{P} 7$ & 3.34 & 17.33 & 1.696 & 3.71 & 750.58 & 403.18 & 590.00 & 78.00 & 110.00 & 1.86 \\
\hline \multirow[t]{2}{*}{ Range } & $2.57-$ & $14.40-$ & $1.14-$ & $2.22-$ & $369.35-$ & $203.44-$ & $448.33-$ & 70.67- & $106.33-$ & $1.70-$ \\
\hline & 3.76 & 17.96 & 1.74 & 4.50 & 914.36 & 440.47 & 701.67 & 81.67 & 118.00 & 2.74 \\
\hline Mean & 3.37 & 16.04 & 1.54 & 3.07 & 587.57 & 264.90 & 593.01 & 77.00 & 112.39 & 2.23 \\
\hline Std dev & 0.23 & 0.80 & 1.07 & 0.61 & 132.22 & 60.35 & 53.11 & 2.44 & 2.90 & 0.23 \\
\hline
\end{tabular}

P1is O-9897, P2 is O-795, P3 is JRO-524, P4 is Acc. 2381, P5 is Acc. 3423, P6 is Acc. 3438, P7 is Acc. 3533 and P8 is Acc. 3860 
Table 4. Correlations between yield and important yield contributing characters of Tossa jute

\begin{tabular}{|c|c|c|c|c|c|c|c|c|c|c|}
\hline & $\mathrm{PH}$ & $\mathrm{BD}$ & GB & GWW & FW & SW & FS & DF & $\mathrm{DM}$ & $\mathrm{Fw} / \mathrm{Sw}$ \\
\hline PH & - & & & & & & & & & \\
\hline BD & -0.021 & - & & & & & & & & \\
\hline GB & $0.304 *$ & 0.235 & - & & & & & & & \\
\hline GWW & $0.313 *$ & $0.375^{* *}$ & $0.467^{* *}$ & - & & & & & & \\
\hline FW & $0.266^{*}$ & $0.389^{* *}$ & $0.495^{* *}$ & $0.762^{* * *}$ & - & & & & & \\
\hline SW & 0.143 & $0.470^{* *}$ & $0.512^{* *}$ & 0.793 & $0.910^{* *}$ & - & & & & \\
\hline FS & 0.047 & 0.045 & 0.188 & 0.105 & 0.009 & 0.023 & - & & & \\
\hline DF & -0.019 & -0.087 & -0.165 & 0.048 & -0.084 & -0.058 & 0.055 & - & & \\
\hline DM & 0.023 & 0.134 & 0.072 & 0.212 & 0.188 & 0.112 & 0.012 & -0.025 & - & \\
\hline FW/SW & $0.299 *$ & -0.149 & -0.016 & -0.050 & 0.253 & -0.166 & -0.016 & -0.049 & 0.170 & - \\
\hline
\end{tabular}

$*$ and $* *$ significant at $5 \%$ and $1 \%$ levels of probability, respectively

PH: Plant Height (m), BD: Base Diameter (mm), GB: Green Bark Thickness (mm), GWW: Green Wight without leaves (g), FW: Fiber Weight, SW: Stick weight, FS: Fiber Strength (MPa), DF: Days to Flowering, DM: Days to Maturity and Fw/Sw: Fiber weight vs. Stick weight 
Table 5. Direct and indirect effect path coefficient

\begin{tabular}{lrrrrrrrrrr}
\hline & PH & \multicolumn{1}{c}{ BH } & \multicolumn{1}{c}{ GBT } & GWWS & \multicolumn{1}{c}{ FW } & \multicolumn{1}{c}{ SW } & FS & \multicolumn{1}{c}{ DF } & DM & FWSW \\
\hline PH & -0.0245 & -0.0087 & 0.0171 & -0.0614 & 1.8347 & -0.9808 & 0.0061 & -0.002 & 0.0001 & -0.7305 \\
BH & 0.0005 & 0.4337 & 0.0132 & -0.0733 & 2.7153 & -3.2926 & 0.0048 & -0.0092 & 0.0001 & 0.4675 \\
GBT & -0.0076 & 0.1041 & 0.0552 & -0.0931 & 3.5225 & -3.5729 & 0.023 & -0.0174 & 0.0001 & 0.1461 \\
GWWS & 0.0076 & 0.1605 & 0.0259 & -0.1981 & 5.4306 & -5.5344 & 0.0133 & 0.0051 & 0.0002 & 0.2045 \\
FW & -0.0061 & 0.1605 & 0.0265 & -0.1466 & 7.3386 & -6.3751 & 0.0024 & -0.0123 & 0.0002 & -0.8181 \\
SW & -0.0034 & 0.2038 & 0.0281 & -0.1565 & 6.6782 & -7.0056 & 0.0024 & -0.0061 & 0.0001 & 0.4091 \\
FS & -0.0012 & 0.0173 & 0.0105 & -0.0218 & 0.1468 & -0.1401 & 0.1211 & 0.0051 & 0.0001 & -0.0877 \\
DF & 0.0005 & -0.039 & -0.0094 & -0.0099 & -0.8806 & 0.4203 & 0.0061 & 0.1023 & 0.0001 & 0.3798 \\
DM & -0.0005 & 0.0564 & 0.0039 & -0.0416 & 1.6145 & -0.7706 & 0.0012 & -0.0031 & 0.0011 & -0.7012 \\
FWSW & -0.0061 & -0.0694 & -0.0028 & 0.0139 & 2.0548 & 0.9808 & 0.0036 & -0.0133 & 0.0003 & -2.9218 \\
\hline Residual Effect $=0.08$ & & & & & & & & & &
\end{tabular}

PH: Plant Height (m), BD: Base Diameter (mm), GB: Green Bark Thickness (mm), GWW: Green Wight without leaves (g), SW: Stick weight, FS: Fibre Strength (MPa), DF: Days $t$ 
Fiber strength varies from 448.33 MPa to 701.67 $\mathrm{MPa}$. The $\mathrm{P} 7 \mathrm{xP} 2$ was the weakest and $\mathrm{P} 7 \mathrm{xP} 4$ was the strongest. The second highest fiber strength was recorded for P1 x P4. The hybrids of P4 have most strength of fiber. This is to note that the fiber strength has a wide range for SE of Mean, from 0.3 to 34.0. Sarker et al., (2012) recorded the highest $(10.78 \mathrm{lb} \mathrm{mg-1)}$ strength in the variety O-9897.

Flowering of half of the flowers in Tossa jute is from 70 days to 82 days, but the mean is 77 days. Found that the generation of P8 took the shortest time to flowering. The mode value of standard error of mean was recorded 2.33 and calculated for 7 genotypes out of 8 . Time of 50 $\%$ flowering envisages the resistance to premature flowering. If a jute variety sown before middle of April does not flower prematurely in seedling stage and flowers at least after 100 days, the variety is known as premature flowering resistant variety (Ghosh, 1983). All the C. olitorius varieties except Chinsurah Green (97 days) were premature flowering resistant. Contrary to it, in $C$. capsularis all the varieties were resistant to premature flowering (Das and Kumar 2012). Sowing time and genotype had major effects on the duration of the vegetative and flowering phases (Rahaman 2016).

The days to maturity of Jute was from 106 days to 118 days, and the mean duration to maturity was 112 days. In case of maturity no reportable pattern was found. In fiber crops, fiber is obtained from the vegetative parts; hence improvement in vegetative part is the main concern of plant breeders. Among various characters, plant height and base diameter of the stem are the most efficient indirect selection criteria for high fiber yield in jute (Maiti et al., 1977, Chaudhury et al., 1981). Significant ranges of variability were observed in plant height, base diameter, green bark thickness, fiber weight, stick weight, and green weight without leaves.

\subsection{Correlation coefficient analysis among different characters of Tossa jute}

Interrelationships of the characters and their association with fiber weight in tossa jute were examined and presented in Table 4. Such study would also help to know the suitability of various characters for indirect selection, because selection of one or more-character results in correlated response in several other traits.

From correlation study it was observed that yield of jute had highly significant positive relationship with fiber weight and base diameter, and had significance with bark thickness, stick weight and green bark weight without leaves. Estimates of correlation do not alone provide comprehensive pictures of the direct and indirect influences of each of the characters to the fiber weight as this trait is the resultant product of combined effect of various factors complementing and counter acting (Das and Kumar 2016). This result corroborated with the finding of Sardana et al., 1990 and Islam et al., 2001. Basal diameter and fiber fineness exhibited direct positive effect on fiber weight at genotypic level only while fiber strength and fiber $\%$ revealed direct positive effect on fiber weight at phenotypic level only. However, significant positive correlation was obtained with base weight, fiber diameter, bark thickness and green weight without leaves. Stick weight produced negative direct effect with fiber weight at both phenotypic levels. Similar results were also reported by several authors with Corchorus species (Maiti et al., 1977; Ghosdastidar and Das, 1984; Islam et al., 2004; Akter et al., 2005).

\subsection{Path coefficient analysis}

The results of path coefficient analysis are given in Table 5. Stick weight (6.67 gm) had highest positive direct effect on fiber yield per plant followed by bark thickness. This finding is in agreement with Senapati et al. (2006). Green weight, plant height and base diameter per plant exhibited high and negative direct effects towards fiber yield. Similar findings were reported by Pervin and Haque (2012). But its significant positive correlations with fiber yield per plant indicated that the indirect selection could be made for high yielding jute genotypes 
through most of the characters having positive indirect effects. The residual effect $(\mathrm{R})$ was 0.08 , indicating that there were also some other characters which although not studied but influenced the yield of fiber per plant.

Direct positive effect of plant height on fiber weight per plant was also reported by several authors (Mandal et al., 1980; Chaudhury et al., 1981; Bordoloi and Das, 1999). On the other hand, base diameter, number of nodes per plant and leaf area showed negative direct effect on fiber weight per plant. Chaudhury et al., (1981) and Bordoloi and Das (1999) reported negative direct effect of base diameter at genotypic level. Direct positive effect of green weight without leaves was reported by Akter et al. (2005). Similar result was obtained by Rahman et. al., (2009). Sawarkar et al., (2014) reported green weight, plant height and base diameter per plant exhibited high and negative direct effects towards fiber yield.

\section{Conclusions}

Thus, the result of the investigation suggests that fiber weight, base diameter, bark thickness, stick weight and Green bark weight without leaves would be the selection parameter to produce jute varieties. The Acc.-3860 resulted high production of fibers in hybrids. Considering the different aspects, it was found that the generation of P6, i.e., Acc.-3860 is comparatively batter than others. The research findings suggested that the generation of parental Accession Acc.-3860 may produce desired segregates. Thus, the study recommends further research on Acc.-3860 in different environmental conditions and field trail.

\section{References}

Ahmadizadeh M., Nori A., Shahbazi H., Aharizad S. 2011. Correlated response of morpho-physiological traits of grain yield in durum wheat under normal irrigation and drought stress conditions in greenhouse. African Journal of Biotechnology, 10 (85): 19771-19779.
Ahmed SS., Khatun, R. 1997. Anatomical screening for selection of high yielding tossa jute ( $C$. olitorius L.). Bangladesh Journal of Plant Breeding and Genetics, 10 (1\&2): 9-12.

Akhtar N., Nazir MF., Rabnawaz A., Mahmood T., Safdar ME., Asif M., Rehman A. 2011. Estimation of heritability, correlation and path coefficient analysis in fine grain rice (Oryza sativa L.). Journal of Animal and Plant Science, 21(4): 660664.

Akter N., Islam MM., Yahiya ASM., Newaz MA. 2010. Genetic divergence for fibre and seed characters in tossa jute (Corchorus olitorius L.). Journal of Biosciences 1: 7-12.

Akter N., Mian MAK., Islam MM., Alim MA., Islam MN. 2005. Estimation of genetic parameters, character association and path analysis in jute ( $C$. olitorius L.) germplasm. Bangladesh Journal of Plant Breeding and Genetics, 18 (I): 35-38.

Alam MJ. 2009. Genetic Divergence, variability, correlation and Path Analysis in White Jute (Corchorus capsularis L). MS Thesis. Sher-E-Bangla Agricultural University, Dhaka, Bangladesh. 66 p.

Ali MA. 1984. Correlation and regression studies between fibre yield and some anatomical characters in white jute. Bangladesh Journal of Jute Fibre Research, 9 (1\&2): 25-30.

Azad-ud-doula Prodhan AKM., Rahman ML., Haque MA. 2001. Effect of Water Stresses on Growth Attributes in Jute II. Plant Based Diameter. Pakistan Journal of Biological Science, 4: 660-664.

Billah M., Sikder B., Latif MA., Chowdhury AK. 2015. Stability Analysis of Selected Jute (Corchorus capsularis L.) Genotypes in Saline and Non-saline Soils of Bangladesh. The Agriculturists, 13(2): 2535 
Biswas SK., Debnath MR, Moniruzzaman M., Islam SN., Tareq MZ. 2018. Genetic Variability Study of Tossa Jute (Corchorus olitorius) Genotypes. International Journal of Business, Social and Science Research, 6(3): 52-55.

Bordoloi PK., Das PK. 1999. Variability, character $\mathrm{r}$ association and path analysis in tossajute ( $C$. olitorius L.). Annals of Agri Bio Research, 4 (12): 179-181.

Chaudhury SK, Sinha MK., Singh DP. 1981. Path analysis in tossa jute. Indian Journal of. Agricultural Sciences, 51(11): 772 775.

Dabholkar AR. 1992. Elements of biometrical genetics. Concept publishing company, New Delhi, India. 431 p.

Das A., Kumar D. 2016. Genetic Divergence and Character Association for Yield and Quality Attributing Characters in Tossa Jute (Corchorus olitorius L.). Electronic Journal of Plant Breeding, 7(3): 529-537.

Das A., Kumar K. 2012. Genetic evaluation and characterization of jute (Corchorus spp. L) genotype using DUS parameters. SAARC Journal of Agriculture, 10(2): 147-153 (2012)

Ghosdastidar KK., Das PK. 1984. Selection breeding in olitorius jute. Perspective in cytology and genetics (ed. Manna GK. and Sinha, U.) 4: 563-567.

Ghosh S., Meena K., Sinha MK., Karmakar PG. 2017. Genetic Diversity in Corchorus olitorius Genotypes Using Jute SSRs. Proc. of National Academy of Sciences, India, Section B: Biological Sciences, (July-Sept 2017) 87(3):917-926

Ghosh T. 1983. Handbook on Jute. FAO, Rome. FAO Plant production and protection paper. 51:219

Hossain MD., Hanafi MM., Jol H., Hazandy AH. 2012. Effects of carbon levels on shoot growth and root characteristics of different kenaf (Hibiscus cannabinus L.) varieties grown on sandy bris soil. African Journal of Biotechnology, 11(25): 67036709

Hossain MD., Hanafi MM., Jol H., Jamal T. 2011. Dry matter and nutrient partitioning of kenaf (Hibiscus cannabinus L.) varieties grown on sandy bris soil. Australian Journal of Crop Science, 5(6): 654-659.

Islam MM. 2007. Variability and path coefficient analysis in jute (Corchorus olitorius L.) of indigenous origin. Digital Archive on Agricultural Theses and Journal. $\mathrm{N}^{\circ}$ Catalog: SAU200701_112. Sher-e-Bangla Agricultural University, Sher-E-Bangla Nagar, Dhaka. 66 p.

Islam MM. 2019. Varietal Advances of Jute, Kenaf and Mesta Crops in Bangladesh: A Review. International Journal of Bioorganic Chemistry, 4(1): 24-41, doi: 10.11648/j.ijbc.2019 0401.15

Islam MR., Islam MM., Akter N., Ghosh RK. 2002. Genetic variability and performance of tossa jute. Pakistan Journal of Biological Science, 5(7): 744-745.

Islam MS., Nasreen A., Begum S., Haque S. 2004. Correlated response and path analysis in tossa jute ( $C$ olitorius L.). Bangladesh Journal of Botany, 33 (2): 99102.

Islam MS., Uddin MN., Haque MM., Islam MN. 2001. Path Coefficient Analysis for Some Fibre Yield Related Traits in White Jute (Corchorus capsularis L.). Pakistan Journal of Biological Sciences, 4: 47-49.

Maiti SN., Biswas SK., Sen S. 1977. A comparative study of selection indices in tossa jute. Mysore Journal of Agricultural Sciences, II (I): 17-22.

Mandal BD., Chandra N., Majumder MK., Banerjee SP. 1980. Pathway to fibre yield in jute (Corchorus olitorius L.). Genetica Polonica. 21 (4): 455-460. 
Miller PJ., Williams JC., Robinson HF., Comstock RE. 1958. Estimates of genotypic and environmental variances and covariances in upland cotton and their implications in selection. Agronomy Journal, 50: 126-131.

Majumdar B., Das S, Saha AR., Chowdhury H., Kundu DK., Mahapatra BS. 2013. Improved Retting of Jute and Mesta with Microbial Formulation (Bulletin No. 04 12013). Central Research Institute for Jute and Allied Fibres (ICAR), Barrackpore, Kolkata, 32p

Nayak BK., Baisakh B., Bastia D. 2009. Genetic divergence among the genotypes of tossa jute (Corchorus olitorius L.). Environment and Ecology, 27: 86-88.

Panse VG., Sukhatme PV. 1989. Statistical Methods for Agriculture Workers. Indian Council of Agricultural Researches, New Delhi, $\mathrm{xvi}+38 \mathrm{p}$

Pervin N., Haque GK. 2012. Path coefficient analysis for fibre yield related traits in Deshi jute (Corchorus capsularis L.). International Research Journal of Applied Life Sciences, 1(3): 72-77.

Rabbani MG., Naher MJ., Hoque S. 2012. Variability, Character association and diversity analysis of Ridge Gourd (luffa acutangula Roxb.) genotypes of Bangladesh. SAARC Journal of Agriculture, 10(2): 1-10.

Rahaman M. 2016. Prospects of developing premature flowering resistant tossa jute $(C$. olitorius) variety for early March and onwards sowing. International Journal of Bioresource Science 3(1): 7-15.
Rahman MR., Ali MA., Bhuiyan MSR., Islam MM., Yahiya ASM. 2009. Genetic parameter, Character association and Path Coefficient analysis of some exotic germplasm of Tossa Jute (Corchorus olitorius L.). Journal of Sher-e-Bangla Agricultural University, 3(2): 19-23.

Sardana S., Sasi-Kumer B., Modak D. 1990. Genetic variability, character association and path analysis in jute germplasm. Bangladesh Journal of Botany, 19: 95-97.

Sarker SR., Chowdhury MAH., Mohiuddin KM., Saha BK. 2012. Influence of different levels of potassium on yield and fibre strength of jute. Journal of Agroforestry and Environment, 6 (1): 39-42.

Satheeshkumar P., Saravanan K. 2012. Genetic variability, correlation and path analysis in rice (Oryza sativa L.). International Journal of Current Research, 4: 82-85.

Sawarkar A., Yumnan S., Patil SG., Mukherjee S. 2014. Correlation and path coefficient analysis of yield and its attributing traits in Tossa Jute (Corchorus olitorius L.) The bioscan, 9(2): 883-887.

Senapati S., Nasim Ali MD., Sasmal BG. 2006. Genetic variability, heritability and genetic advance in Corchorus sp. Environment and Ecology, 24S (1): 1-3

Singh RK., Chaudhury BP. 1985. Biometrical method in quantitative genetic analysis (rev. ed.) Kalyani Publishers, New Delhi, India. 318 p. 